Universidad de Lima

Facultad de Psicología

Carrera de Psicología

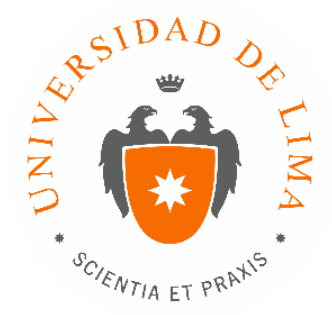

\title{
PROGRAMA DE ORIENTACIÓN VOCACIONAL
}

Trabajo de suficiencia profesional para optar el título profesional de Licenciado en Psicología

\section{Romina Ascenzo Rouillon}

Código 20110091

Lima - Perú

Febrero de 2019 

PROGRAMA DE ORIENTACIÓN VOCACIONAL 


\section{TABLA DE CONTENIDO}

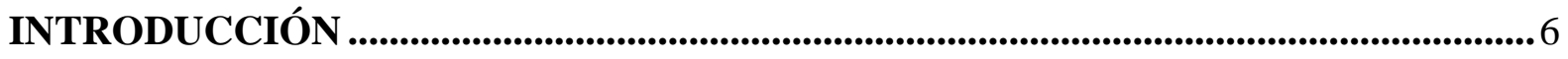

CAPÍTULO I: IDENTIFICACIÓN DEL PROBLEMA ........................................................7

CAPÍTULO II: DESCRIPCIÓN DE LAS ACTIVIDADES REALIZADAS .................... 11

2.1 Previo al proceso de Orientación Vocacional ................................................... 11

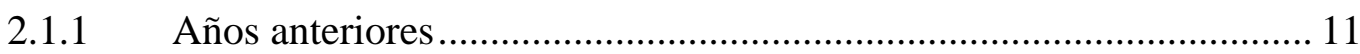

2.1.2 Quinto de secundaria ............................................................. 12

2.2 Propuesta de Orientación Vocacional ......................................................... 12

2.2.1 Evaluación e informes de Orientación Vocacional .................................13

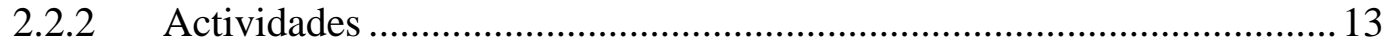

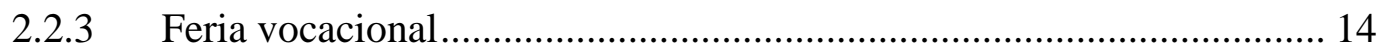

2.2.4 Devolución de resultados...............................................................

2.3 Luego de finalizar el programa de Orientación Vocacional ..............................15

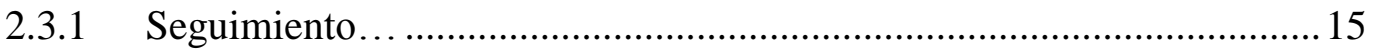

CAPÍTULO III: RESULTADOS DE LA INTERVENCIÓN ............................................16

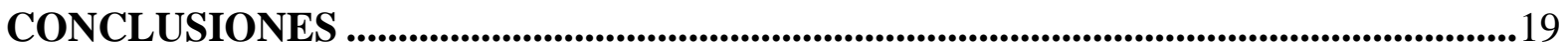

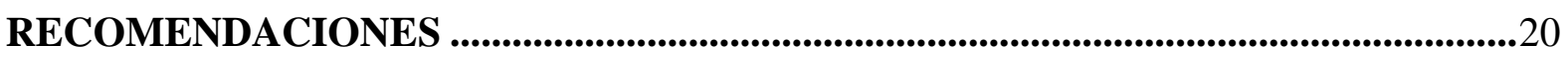

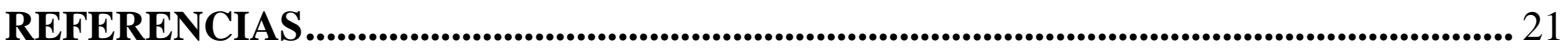

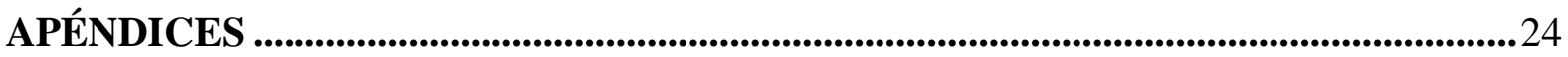




\section{ÍNDICE DE APÉNDICES}

Apéndice 1: Sesión de Orientación Educativa (6to grado de primaria) ................................ 25

Apéndice 2: Presentación del Programa de Orientación Vocacional en Power Point ......... 27

Apéndice 3: Vaceado de datos de la Encuesta sobre Intereses Profesionales.......................29

Apéndice 4: Modelo de Informe de Orientación Vocacional ................................................. 30 


\section{INTRODUCCIÓN}

La Orientación Vocacional es un proceso en el cual se busca guiar a una persona para definir su elección profesional. Para ello, debe contar con algunas sesiones de entrevista y evaluación con un orientador vocacional, en las cuales se le brinda el asesoramiento y herramientas necesarias para lograr identificar sus características personales, tales como: intereses, habilidades, fortalezas, debilidades, y personalidad, las cuales le permiten definir con mayor claridad sus opciones (Rodríguez, 2013).

Se considera de vital importancia llevar a cabo este proceso en la etapa previa a la elección vocacional debido a que este permite: sensibilizar a los jóvenes sobre la importancia del proyecto de vida, conocer la realidad del mercado laboral, aclarar dudas ocupacionales, promover la toma de decisiones, motivar a los jóvenes al ingreso y mantener la continuidad académica, y los educa en cuanto a los factores de riesgo. (Correa, 2015; Ministerio del Trabajo y Promoción del Empleo, s.f).

Si bien este proceso siempre fue importante, años atrás las personas solo buscaban orientadores vocacionales cuando se les presentaban fracasos laborales o de elección de carrera. Hoy en día, se sabe que este ha cobrado mayor relevancia y posee un valor más preventivo, con el fin de reducir la incertidumbre que surgen en esta etapa y las posibilidades de enfrentar fracasos vocacionales (Phutura Jóvenes, 2018).

Con el presente trabajo se busca contextualizar el proceso de Orientación Vocacional en la actualidad $\mathrm{y}$ enfocarse en un programa realizado en un colegio privado femenino. Considerando la identificación del problema, las actividades realizadas, los resultados encontrados, las conclusiones y recomendaciones. 


\section{CAPÍTULO I: IDENTIFICACIÓN DEL PROBLEMA}

En el último año de colegio, los alumnos se encuentran por dar inicio a una nueva etapa muy importante en su vida: la elección vocacional. Para algunos, esto puede ser muy sencillo debido a que tienen una decisión ya tomada; sin embargo, para otros, esto no es fácil, volviéndose una fase complicada y estresante (Aragón, 2014).

Si bien este periodo siempre ha sido complejo, en los últimos años esto se ha incrementado para los estudiantes a consecuencia del entorno cambiante en el que viven. A partir de los diferentes avances científicos y tecnológicos que se han desarrollado, así como las nuevas condiciones sociales y económicas que se generan, las personas se han visto expuestas a una gran cantidad de cambios e información, así como a una mayor accesibilidad a la misma. Todo esto trae consigo grandes transformaciones en la vida de las personas: nuevas necesidades, diversidad en la oferta educativa (un aumento de casas de estudio y profesiones), mayores oportunidades para estudiar en el extranjero, entre otros; a diferencia de lo que ocurría años atrás, cuando tenían menos opciones de carreras y centros de estudio, por lo que se podría considerar como una situación "más fácil” debido a que las opciones eran más limitadas (Alonso, Casado, López Lugones y Sánchez, 2014; Rodríguez, 2013).

A esto se añade el hecho de que los estudiantes toman la decisión vocacional estando en un tiempo crítico para ellos: la adolescencia (Aragón, 2014). El paso entre la niñez y la adultez trae consigo grandes cambios en diferentes aspectos de su vida. En lo físico, el desarrollo de la madurez biológica y neurológica; en lo social, el aumento de las relaciones íntimas y significativas con sus pares; y por último, en el psicológico, se desarrolla y consolida su identidad (Iglesias, 2013; Musitu y Callejas, 2017).

Debido a que los cambios evolutivos ocurren hasta los veintiún años, aproximadamente, su proceso de desarrollo y autoconocimiento está incompleto, por lo que los jóvenes aún no se 
encuentran lo "suficientemente preparados" para su elección (Alonso et al., 2014; Iglesias, 2013; Rodríguez, 2013).

Sumado a todo esto, también hay que considerar las situaciones y preocupaciones que enfrentan los estudiantes en este momento: culminar de manera satisfactoria el último año de colegio, el temor a equivocarse y fracasar académicamente, el ingreso a la universidad, el temor a lo desconocido y el asumir nuevas responsabilidades (Universia, 2011). Adicionalmente, Alonso et al. (2014) indican que se debe considerar el hecho de que la educación hoy en día ya no ofrece garantías de salida laboral.

Elegir una carrera por seguir los deseos de los padres o la tradición familiar, por influencia de sus amistades, porque consideran que "está de moda", que es "fácil", que tendrán un gran ingreso económico o solo porque quieren, o no, llevar ciertos cursos, son algunos de los errores comunes que cometen los estudiantes al momento de elegir una profesión. Estos factores podrían llevarlos al fracaso académico y frustración, y, a largo plazo, a la insatisfacción, influyendo en la calidad de su trabajo, disminuyendo así su productividad y contribuyendo a que no haya un clima laboral adecuado (Rodrigo, 2018).

Para aminorar el efecto de estas dificultades y evitar los errores anteriormente mencionados, se deben considerar ciertos elementos en el proceso de la elección vocacional y proyecto de vida. Hay que tomar en cuenta, en primer lugar, los intereses, es decir, tener claridad en cuanto a qué es lo que le gusta hacer y lo que no. Segundo, las habilidades, reconocer qué es lo que sabe y lo que le cuesta más hacer. Tercero, la personalidad, conocerse a profundidad tomando en cuenta sus fortalezas y debilidades, ya que estas favorecen o, por el contrario, pueden ser contraproducentes en el desempeño de ciertas actividades. Cuarto, el aspecto cognitivo, reconocer sus recursos y áreas de mejora. Quinto, los aspectos de la realidad, que incluyen tomar en cuenta su situación socioeconómica, personal y familiar para considerar sus opciones. Por último, las expectativas a futuro, saber qué es lo que buscan lograr, lo que 
esperan en una carrera y lo que pretenden obtener con ella (Rodríguez, 2013; Vivas, 2015). El conocimiento de todos estos elementos y tomarlos en cuenta en el momento de la elección, les permite disminuir la lista de opciones vocacionales y tener mayores probabilidades de éxito profesional.

Al analizar el proceso de elección vocacional en el Perú, se observa que más del $70 \%$ de los estudiantes que han culminado el colegio no sabe cuál es la carrera que va a estudiar (“Jóvenes que terminan el colegio no saben qué carrera estudiar”, 2014). Asimismo, Ramos (2003) y Logros indican que el $80 \%$ de los jóvenes que postulan a la universidad lo hacen con poco conocimiento de la carrera en la que se inscriben, realizándolo solo por lo que ésta representa socialmente (como se citó en Mori, 2012).

Según Logros, uno de los principales problemas en la educación es la deserción universitaria, la cual alcanza una tasa del 17\% (como se citó en Mori, 2012). Son entre cuarenta y cincuenta mil jóvenes los que abandonan sus estudios universitarios cada año (generando una gran pérdida económica para los padres de familia), de los cuales el $70 \%$ corresponde a estudiantes de universidades privadas y el 30\% restante a las estatales (Plasencia, 2011). Asimismo, Penta Analytics (2017) indica que el 27\% de los ingresantes a universidades privadas abandonan su carrera en el primer año de estudios, y esta cifra aumentaría a $48 \%$ si es que se toma en consideración la cantidad de alumnos que no terminan una carrera. Según una investigación realizada por el economista Gustavo Yamada, el 60\% de egresados refiere que si pudieran cambiarían de carrera, y reconocen que la mala decisión sería por falta de orientación adecuada (“Egresados universitarios cambiarían de carrera”, 2015).

La falta de Orientación Vocacional en los alumnos y la consecuente equivocación en la elección de la carrera universitaria no solo llevaría al cambio de ésta y a la insatisfacción laboral, sino que tiene repercusiones sobre la salud física y mental de los jóvenes, pudiendo generar estrés y ansiedad en ellos (Career Hunter, 2017; Kunnen, 2015). 
Con lo expuesto anteriormente, se puede evidenciar la importancia de la intervención en este ámbito, la cual implica un proceso transversal que ocurre a lo largo de la vida de las personas, permitiendo que los alumnos lleguen a quinto de secundaria más preparados y seguros al momento de elegir su profesión (Ministerio de Educación del Perú [MINEDU], 2013). En esta intervención se brinda orientación enfocada en el autoconocimiento, herramientas para la búsqueda y análisis de información sobre las carreras y centros de estudio, la contextualización de las expectativas de los alumnos y espacio de contención (Paoli, 2018). En el presente trabajo se expondrá el programa de Orientación Vocacional llevado a cabo por el Departamento Psicopedagógico de un colegio de Lima. La institución, católica y de gestión privada, cuenta con una población estudiantil femenina de ochocientos cuarenta alumnas aproximadamente, las cuales cursan entre quinto grado de primaria a quinto grado de secundaria. 


\section{CAPÍTULO II: DESCRIPCIÓN DE LAS ACTIVIDADES Y TAREAS REALIZADAS}

A continuación, se presentarán las actividades y tareas llevadas a cabo por el Departamento Psicopedagógico para el proceso de Orientación Vocacional.

\subsection{Previo al proceso de Orientación Vocacional}

En este colegio se trabaja el proceso de Orientación Educativa desde primaria, por lo que se presentarán las actividades llevadas a cabo desde esos años, así como lo trabajado en quinto de secundaria previo al programa de Orientación Vocacional.

\subsubsection{Años anteriores}

Desde quinto y sexto de primaria se realizan actividades sobre Orientación Educativa con las estudiantes. En estas, se busca que las alumnas se involucren e investiguen sobre las profesiones que encuentran dentro de los miembros de su familia, reconozcan sus gustos y fortalezas, describan cómo se ven en el futuro, entre otros (ver apéndice 1), para que vayan tomando conciencia de sus gustos e intereses y formen una base que les permita tomar una decisión segura en el futuro.

A partir de tercero de media, las alumnas empiezan a asistir a las ferias vocacionales con el propósito de brindarles información y que empiecen a prepararse para el futuro.

A finales de cuarto de secundaria, se trabaja el Programa de Orientación Vocacional "Yo Decido mi futuro", ejecutado por la Universidad Peruana de Ciencias Aplicadas (UPC). En este, se realizan parte de las evaluaciones requeridas para el desarrollo de los informes de orientación. Dentro de este se evaluó: el Test de Intereses Profesionales, el cual refleja el gusto por 
realizar ciertas actividades, la habilidad para aprenderlas y la satisfacción que consideran brindará el hacerlas como su profesión; Test de Preferencias del Temperamento, que muestra las características más importantes de la personalidad en cuatro dimensiones; y el Test de Talentos, que permite identificar los talentos de cada uno, para poder desarrollarlos y llevarlos al éxito en su futuro profesional. Las pruebas son elaboradas y estandarizadas por Effectus Fischman Consultores. Los resultados de la evaluación fueron entregados al colegio durante el primer trimestre del siguiente año.

\subsubsection{Quinto de secundaria}

A principio de año, se destinaron dos sesiones de tutoría para brindar información sobre el proceso de orientación. En la primera, se explicaron las características del proceso, así como de las actividades del programa que se trabajaría en el colegio (ver apéndice 2). La segunda, fue una sesión abierta, para responder a las preguntas de las alumnas y brindarles un espacio de contención, ya que tenían muchas inquietudes sobre el último año de colegio y la decisión vocacional.

Por otro lado, durante el primer trimestre, se entregó a las alumnas una encuesta con preguntas abiertas y cerradas, para que indiquen las carreras y universidades que más les interesaban, a nivel nacional e internacional. Luego, se hizo un vaciado de datos con esta información para considerarla al organizar el programa de orientación (ver apéndice 3).

\subsection{Programa de Orientación Vocacional}

A continuación, se describirán las tareas que se realizaron para cumplir con los objetivos del programa. En primer lugar, aplicar las pruebas, elaborar los informes y realizar la devolución de resultados a la totalidad de las alumnas, así como 
contar con la asistencia de toda la promoción a la feria vocacional. En segundo lugar, brindar orientación, asesoramiento, y dotar de herramientas informativas, buscando apoyarlas durante el proceso y a elegir de manera informada y segura.

\subsubsection{Evaluación e informes de Orientación Vocacional}

Para completar las evaluaciones realizadas en el programa "Yo Decido mi futuro”, el Departamento Psicopedagógico evaluó el área cognitiva con el P.M.A. (Test de Aptitudes Mentales Primarias) de Ugarriza y Palma (1999). Luego, fue corregido e interpretado, para ser integrado en al informe de orientación. Este consistía en un resumen de los resultados brindados por la UPC integrando los resultados del P.M.A.

\subsubsection{Actividades}

A lo largo del año, se realizaron diversas actividades que permitieron a las alumnas tener mayor información, y, a su vez, un mayor acercamiento a las carreras de su interés. Para ello, se buscó contar con universitarios y profesionales que fueran un modelo de referencia cercano para generar un mayor impacto en ellas.

La primera actividad fue el "Conversatorio con profesionales", el cual se llevó a cabo con ocho padres de familia del colegio de diferentes profesiones. Los conversatorios fueron breves para que los padres pudieran pasar por todos los salones y que las alumnas pudieran tener la experiencia de escuchar un poco de cada uno de ellos y resolver sus dudas.

La segunda actividad fue "Compartiendo experiencias" se contó con la participación de cinco exalumnas de los últimos años, para que dieran testimonio sobre su reciente experiencia universitaria y laboral. 


\subsubsection{Feria vocacional}

El Departamento Psicopedagógico decidió reestructurar la tradicional feria vocacional que lleva a cabo todos los años para ahora incluir nuevas actividades, las cuales se realizaron por grupos en simultáneo. Esto con el fin de brindar una mayor diversidad de fuentes de información, para convertirlo en un espacio entretenido y enriquecedor para las alumnas. Tomando en cuenta la información recogida en la encuesta aplicada, se realizaron talleres en los que expositores de las distintas universidades hablaban sobre las carreras más relevantes para las alumnas. Para esta actividad, las alumnas se inscribían en las dos carreras que más les llamara la atención.

Además, se llevaron a cabo charlas de distintas universidades con actividades que les brindaban estrategias y les permitían trabajar sobre su elección vocacional como visualizarse a futuro, reflexionar sobre ellas mismas, entre otras.

Por último, se realizaron exposiciones propias de la feria. Se contó con una gran cantidad de universidades e institutos, nacionales e internacionales, las cuales resolvían las dudas de las estudiantes y brindaban información sobre las mallas curriculares, el modo de admisión, beneficios y de su prestigio.

Al finalizar, se les entregó a las alumnas una encuesta de satisfacción para evaluar todas las actividades a las que habían asistido.

\subsubsection{Devolución de resultados}

Debido a la cantidad de alumnas, ciento veinte aproximadamente, y al tiempo con el que se contaba, se realizaron entrevistas semiestructuradas 
(Fernández Ballesteros, 2011) con grupos pequeños, de cuatro a cinco alumnas, para hacer la devolución de resultados. Se le entregó a cada una su informe de Orientación Vocacional y se les explicando paso a paso el objetivo de cada prueba aplicada y el significado de los resultados obtenidos (ver apéndice 4). Luego, cada una revisaba sus resultados y hacía preguntas en caso algo no hubiera quedado claro. Al finalizar con todo el informe se les preguntaba cómo se sentían con los resultados obtenidos y se brindaba un espacio para dialogar y compartir si es que consideraban que estos iban de acuerdo a lo que ellas pensaban. Por último, se conversó sobre su proceso de elección, si ya tenían una decisión tomada, habían reducido sus opciones de carreras o universidades; teniendo un registro de sus respuestas y permitiendo brindar mayor atención a las alumnas que tuvieran mayores inquietudes.

\subsection{Luego de finalizar el programa de Orientación Vocacional}

\subsubsection{Seguimiento}

De acuerdo a la información obtenida en las entrevistas grupales, durante el último trimestre se realizaron entrevistas personales semiestructuradas (Fernández Ballesteros, 2011) para hacer seguimiento a las alumnas que tenían mayor dificultad para decidir sobre su profesión o las que pedían conversar con las psicólogas, dándoles mayor acompañamiento y herramientas para la búsqueda de información y toma de decisiones. 


\section{CAPÍTULO III: RESULTADOS DE LA INTERVENCIÓN}

A partir de la ejecución del programa de Orientación Vocacional, se cumplieron los objetivos planteados. En primer lugar, se logró aplicar tanto las pruebas del programa "Yo Decido mi futuro" como el P.M.A al $100 \%$ de las alumnas, lo que permitió realizar los informes de Orientación Vocacional y la devolución de resultados a la totalidad. A pesar de no contar con un registro detallado de la asistencia a la feria vocacional, se confirmó que todas las estudiantes que asistieron ese día al colegio estuvieron en la feria. En segundo lugar, durante las entrevistas realizadas, las alumnas indicaron que se sintieron apoyadas a lo largo del proceso. Además, se identificó que se sintieron más tranquilas y seguras al conocer que los resultados obtenidos en el informe se alineaban a lo que ellas pensaban. Asimismo, se pudo observar que las alumnas fueron agentes activos durante este proceso, ya que se evidenció su interés y búsqueda de información constante, tomando decisiones a conciencia según sus preferencias, lo que permitió que antes de finalizar el año escolar gran cantidad de ellas haya ingresado a la universidad, o tuviera la decisión tomada. Las pocas alumnas que aún no lo habían conseguido, habían reducido sus opciones a dos o tres alternativas de carrera o centro de estudio.

Asimismo, fue clave para el cumplimiento de los objetivos: la organización, la cual permitió contar con una planificación con pasos a seguir claros, así como con tiempos y momentos para la realización de las actividades de manera anticipada. Un ejemplo de esto fue la aplicación temprana de los instrumentos de medición, que permitió fijar nuevas fechas para evaluar a las alumnas que se ausentaron, y aún contar con el tiempo necesario para elaborar los informes sin afectar las fechas de entrega previamente establecidas. Y el trabajo en equipo, el cual se sintió por parte de todo el profesorado con su disposición para ayudar con lo que fuera necesario. Por ejemplo, hubo una gran disposición por parte del personal cuando 
se realizó la feria vocacional, en la que se desarrollaban todas las actividades en simultáneo y se requería de una gran cantidad de personas comprometidas para hacerlo, así mismo, los profesores se mostraron flexibles ante las necesidades que pudieran surgir al llevar a cabo el programa (como sacar alumnas de su clase, cambios de horarios, etc.).

El desarrollo de este programa logró que las alumnas tuvieran un conocimiento más profundo sobre ellas mismas, así como que presenciaran y analizaran una gran cantidad de fuentes de información con respecto a diferentes carreras y universidades, permitiéndoles estar más seguras y empoderas para la elección vocacional (Paoli, 2018; Rodríguez, 2013; Vivas, 2015).

La estructura y modo de trabajo del programa permitieron contar con una población segura, informada y preparada para la elección profesional, lo que contribuiría a disminuir los cambios de carrera, el arrepentimiento de la profesión estudiada y la deserción universitaria ("Egresados universitarios cambiaría de carrera", 2015; Penta Analitycs, 2017). Además, a futuro, se reduciría el número de personas que no se encuentran satisfechas laboralmente, lo que mejoraría la productividad en las empresas y disminuiría los problemas, tanto de salud física como mental, que presentan los jóvenes (Career Hunter, 2017; Kunnen, 2015; Rodrigo, 2018).

Este tipo de programa podría ser replicado en diferentes instituciones educativas, tomando en consideración la realidad en la que se encuentran los estudiantes y sus características, para conocer cuáles serían las opciones más adecuadas para ellos.

Si bien el programa fue considerado como positivo, se encuentran ciertos aspectos en los que se podría mejorar para los próximos años. En primer lugar, la ausencia de algunas alumnas hizo que se tomara más tiempo del previsto en la etapa de aplicación de pruebas porque debía programarse una nueva fecha. En segundo lugar, en ocasiones fue complicado entrevistar a todas las estudiantes en los tiempos programados, pues no podían perder algún curso o 
trabajo, lo que demoraba un poco más el proceso de devolución de resultados. En tercer lugar, con la encuesta aplicada sobre las actividades realizadas en la feria vocacional, se observó que algunas no fueron agrado de las estudiantes como se esperaba. En cuarto lugar, el programa no contaba con metas claras para conocer la cantidad o el porcentaje de alumnas que se esperaba haya tomado la decisión vocacional o ingresado a la universidad al finalizar al año, lo que dificulta su evaluación cuantitativa. En quinto lugar, el programa no cuenta con una estrategia para monitorear cómo les va a las exalumnas en los siguientes años, por lo que no se puede evaluar los beneficios del programa a largo plazo. 


\section{CONCLUSIONES}

- La metodología empleada en el programa de Orientación Vocacional permitió la consecución de todos los objetivos esperados.

- Es imprescindible considerar la Orientación Vocacional como un proceso que ocurre a lo largo de la vida de las personas, para que tomen la elección profesional estando preparadas e informadas.

- El programa de orientación debe contar con objetivos claros, cuantitativos y cualitativos, para poder monitorear el desarrollo de estos y realizar las modificaciones necesarias si es que estos no se cumplen.

- Es importante que el psicólogo encargado del proceso de orientación cree un clima de empatía y confianza, en el cual brinde soporte y herramientas que permitan a las estudiantes afrontar este periodo de cambios e inestabilidad en su vida de la mejor manera posible.

- Se identifica la carencia de un proceso evaluativo en el programa de Orientación Vocacional en el cual se puedan medir los resultados del programa a largo plazo, para verificar si estos han sido adecuados y beneficiosos o no.

- La evaluación de los alumnos con cada una de las áreas involucradas en el proceso vocacional permitirá que los informes, y, por lo tanto, la orientación, sea íntegra y beneficiosa para ellos. 


\section{RECOMENDACIONES}

A continuación, se detallarán las recomendaciones:

- Para ir preparándolos y creando expectativa en los alumnos, se recomienda trabajar el proceso de Orientación Vocacional desde los primeros años de escolaridad, con actividades que sean atractivas y vayan de acuerdo a la edad de los estudiantes, siguiendo lo planteado por el MINEDU (2013).

- Plantear objetivos cuantitativos y cualitativos claros desde el inicio del programa para poder guiar, monitorear y hacer modificaciones en este en el momento en que se considere necesario. Así como para hacer comparaciones según años anteriores y proyectarse a obtener cada vez mejores resultados.

- Se aconseja que el psicólogo tenga momentos para compartir con los alumnos, en los que exprese su interés por ellos, haciéndolos sentir comprendidos, apoyados y valorados, lo cual genera una red de apoyo positiva necesaria para los estudiantes en estos momentos.

- Realizar una encuesta virtual a las exalumnas en la que se evalúe los resultados del programa de Orientación Vocacional a largo plazo, en la cual se presenten preguntas sobre su situación actual, permitiendo conocer: el grado de satisfacción, cambios de carrera, deserción universitaria, entre otros aspectos.

- Se aconseja contar un cronograma de actividades en el que se programen estas con anticipación, para tener la posibilidad de hacer reprogramaciones en caso sea necesario. Para disminuir así la posibilidad de tener alumnos que cuenten con evaluaciones incompletas por falta de tiempo. 


\section{REFERENCIAS}

Alonso, N., Casado, S., López, N., y Sánchez, Andrea. (2014). La elección vocacional en una sociedad cambiante. Reflexión Académica en Diseño y Comunicación, 15(22). Recuperado de https://fido.palermo.edu/servicios_dyc/publicacionesdc/vista/detalle_articulo.php?id_1 ibro=483\&id_articulo=10116

Aragón, R. (2014). Orientación Vocacional en adolescentes [mensaje en un blog]. Recuperado de https://www.avanza-psicologia.es/orientacion-vocacionaladolescentes/

Career Hunter. (14 de febrero del 2017). El riesgo de elegir la carrera equivocada. Recuperado de https://www.prnewswire.com/news-releases/the-shocking-cost-ofmaking-a-wrong-career-choice-613686493.html

Correa, J. O. (22 de junio del 2015). ¿Qué es la Orientación Vocacional? Recuperado de https://www.ucn.edu.co/virtualmente/bienestarCblog/Lists/EntradasDeBlog/Post.aspx?ID=5

El 60\% de egresados universitarios cambiaría de carrera. (26 de julio del 2015). $R P P$. Recuperado de http://rpp.pe/lima/actualidad/el-60-de-egresados-universitarioscambiaria-de-carrera-noticia-820577

Fernández Ballesteros, Rocío. (2011). Evaluación Psicológica: Conceptos, métodos y estudio de casos. Madrid: Pirámide

Iglesias, J. L. (2013). Desarrollo del adolescente: aspectos físicos, psicológicos y sociales. Pediatría Integral, 17(2), 88-93. Recuperado de https://www.adolescenciasema.org/wp-content/uploads/2015/07/Desarrollo-deladolescente.pdf

Kunnen, E. (2015). The effect of a career choice guidance on self-reported psychological problems. Frontiers in Psychology, 5, 1-8. Recuperado de https://www.ncbi.nlm.nih.gov/pmc/articles/PMC4045055/pdf/fpsyg-05-00547.pdf 
Más del 70\% de jóvenes que termina el colegio no sabe qué carrera estudiar. (24 de junio del 2014). Gestión. Recuperado de https://gestion.pe/tendencias/70-jovenes-terminacolegio-carrera-estudiar-63825

Ministerio de Educación del Perú (2013). Tutoría y Orientación Educativa. Recuperado de http://tutoria.minedu.gob.pe/assets/cartilla-orientacion-vocacionali.pdf?fbclid=IwAR1j81z0djFKcSyqwfjTjnxM1D6JpgDw1be2R6f1dhCi1zHgX9O3sN m5big

Ministerio de Trabajo y Promoción del Empleo (s.f). La importancia de la Orientación Vocacional. Recuperado de https://www.trabajo.gob.pe/archivos/file/dnpefp/SOVIO/articulos_interes/ARTICUL O_DE_INTERES_1.pdf

Mori, M. (2012). Deserción universitaria en estudiantes de una universidad privada de Iquitos. Revista Digital de Investigación en Docencia Universitaria, 6(1). Recuperado de http://www3.upc.edu.pe/html/0/boletines/ridu/articulo-4-desercion-estudiantilmori.pdf

Musitu, G., y Callejas J. (2017). El Modelo de Estrés Familiar en la Adolescencia: MEFAD. doi: https://doi.org/10.17060/ijodaep.2017.n1.v1.894

Paoli, G. (05 de junio del 2018). ¿Qué es la Orientación Vocacional? [mensaje en un blog]. Recuperado de https://www.gabrielapaoli.com/que-es-la-orientacion-vocacional/

Penta Analytics. (18 de julio del 2017). En el Perú, el 27\% de los ingresantes a universidades privadas abandona su carrera en el primer año de estudios. Recuperado de http://www.analytics.cl/peru-27-los-ingresantes-universidades-privadas-abandonancarrera-primer-ano-estudios/

Phutura Jóvenes (24 de abril del 2018). ¿Por qué la orientación vocacional es esencial en los escolares? Recuperado de https://www.phuturaejecutivo.com/blog/orientacionvocacional-esencial-escolares/

Rodrigo, B. (27 de abril del 2018). Errores comunes que debes evitar a la hora de elegir carrera. Recuperado de https://www.abc.es/economia/abci-errores-comunes-debesevitar-hora-elegir-carrera-201804251959_noticia.html 
Rodríguez, M. (4 de febrero del 2013). Elección Vocacional y Crisis de la Adolescencia [mensaje en un blog]. Recuperado de http://blog.pucp.edu.pe/blog/perfil/2013/02/04/eleccion-vocacional-y-crisis-de-laadolescencia/

Ugarriza, N., y Palma, S. (1999). Estandarización del Test de Aptitudes Mentales Primarias (PMA) en escolares de Lima Metropolitana. Scientia, 1(1), 97-112.

Universia. (30 de marzo del 2011). ¿Estrés por la elección de carrera? Afróntalo. Recuperado de http://noticias.universia.edu.pe/en-portada/noticia/2011/03/30/806564/estreseleccion-carrera-afrontalo.html

Vivas, G. (2015). Factores causales del cambio de la carrera en estudiantes universitarios que al volver a elegir optaron por licenciatura en Psicopedagogía en la Universidad Abierta Interamericana (Tesis para optar el título profesional de Licenciado en Psicopedagogía). Universidad Abierta Interamericana. Recuperado de http://imgbiblio.vaneduc.edu.ar/fulltext/files/TC120323.pdf 
APÉNDICES 


\section{APÉNDICE 1: Sesión de Orientación Educativa (6to grado de primaria)}

Sexto grado de Primaria - Tutoria

Nombres y apellidos:

Sección:

\section{PROYECTO DE VIDA}

1. ¿Quién soy?
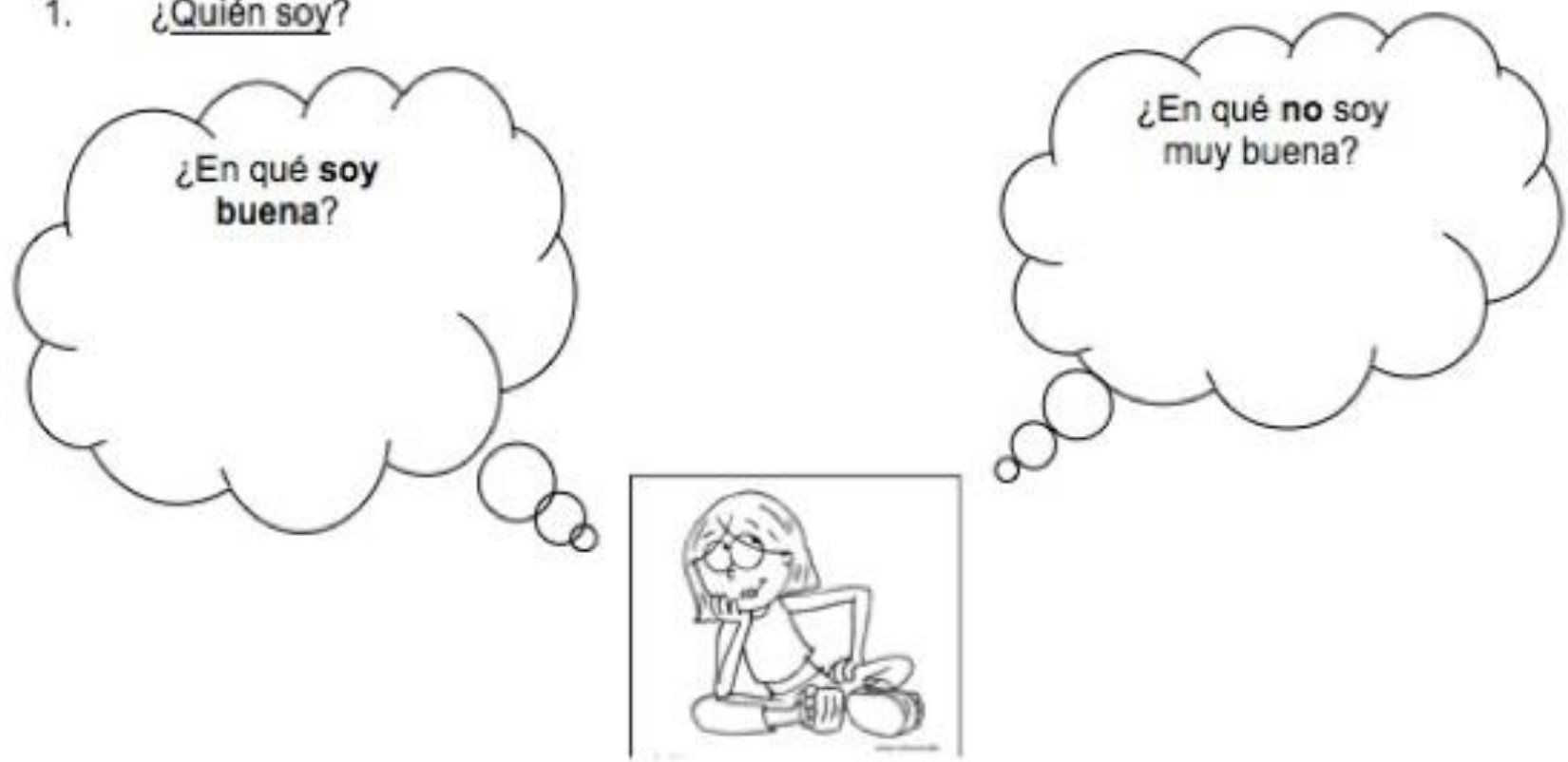

2. ¿Qué cosas me encantaba hacer cuando yo era pequeña?
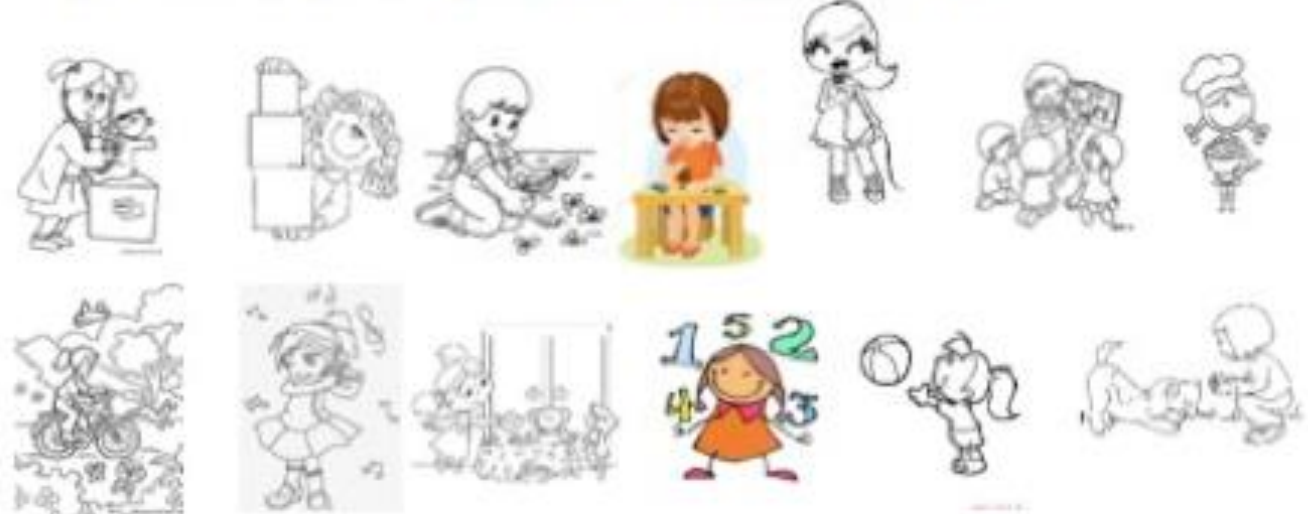
3. ¿Cómo me visualizo a mi misma a los 26 años?
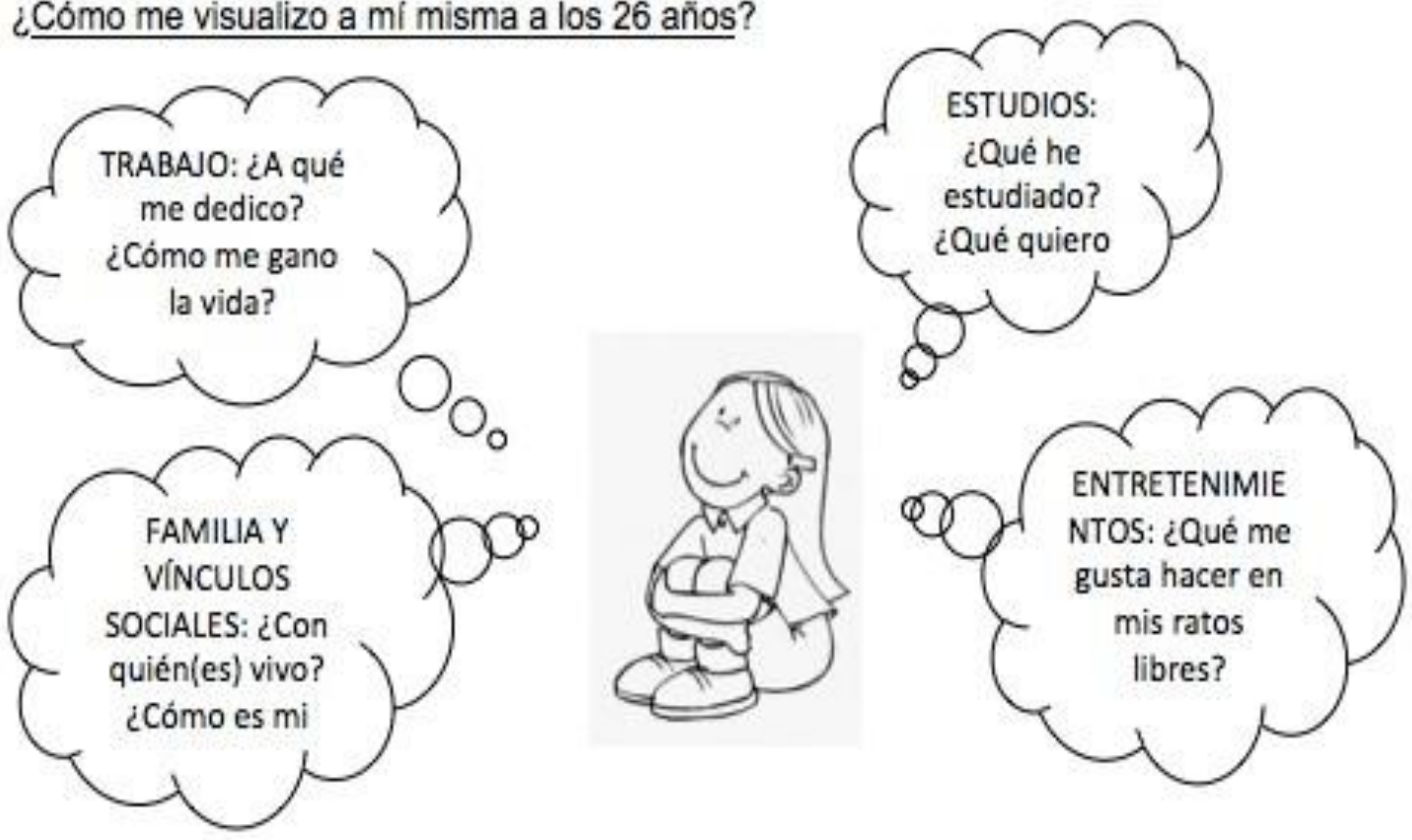

4. ¿Qué puedo ir haciendo desde ahora para lograr mis metas?

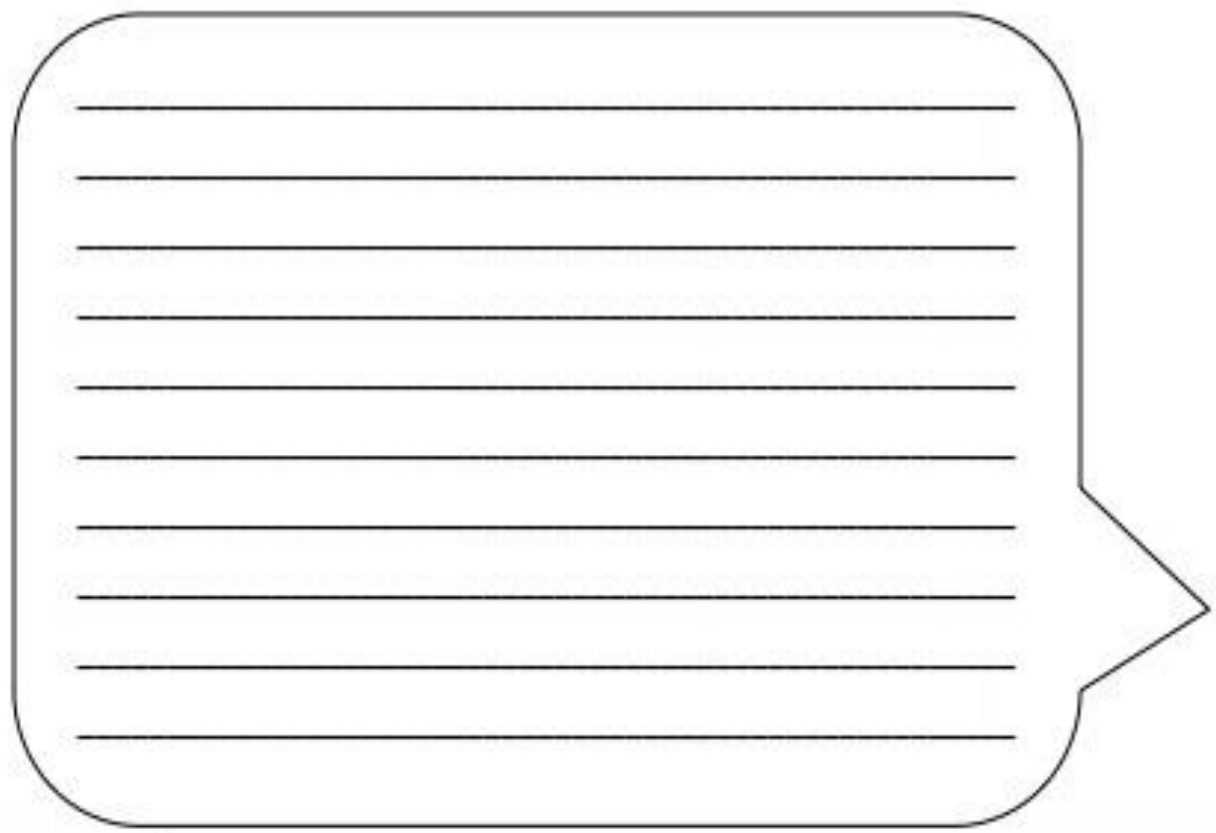




\section{APÉNDICE 2: Presentación del Programa de Orientación}

\section{Vocacional en Power Point}
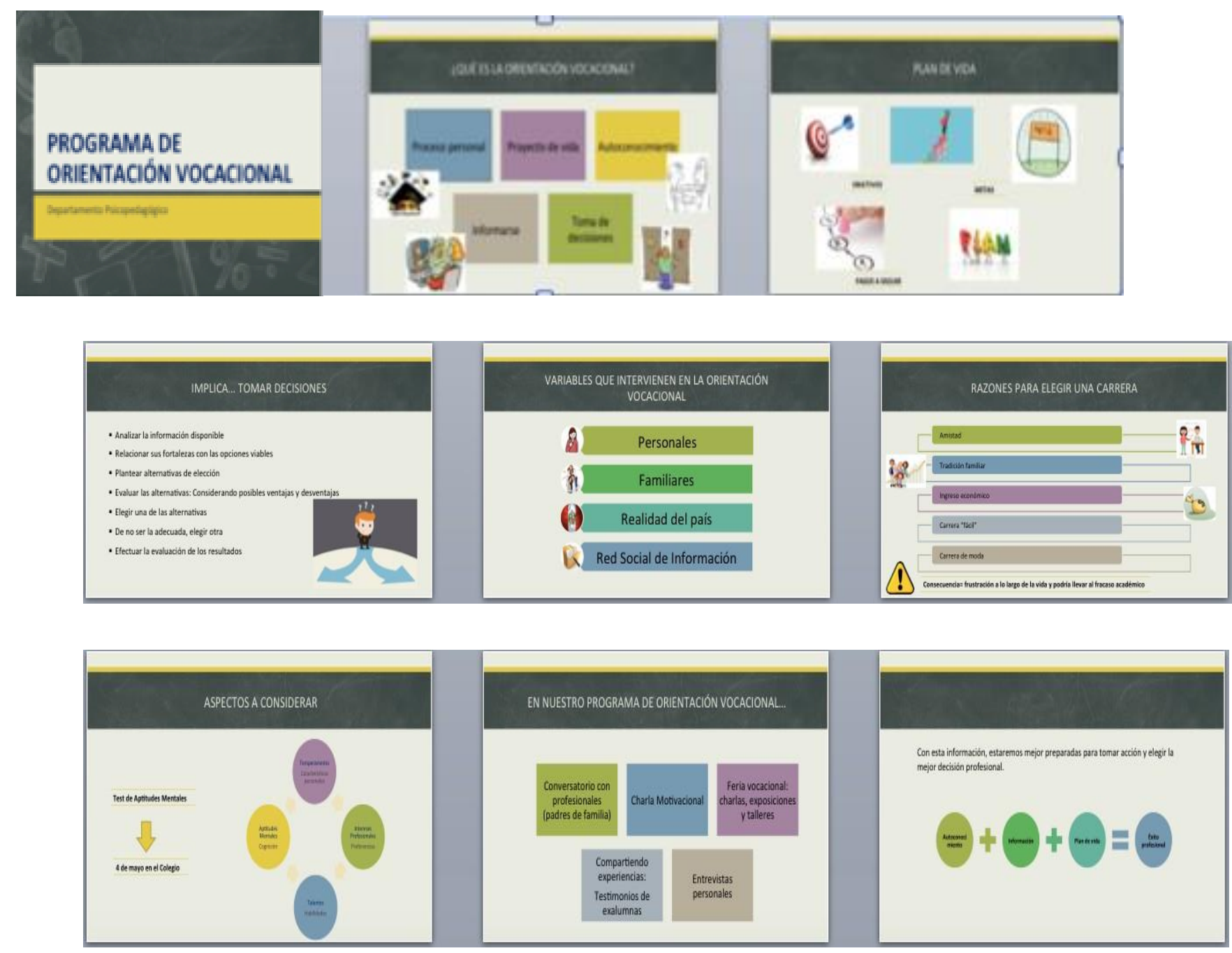


\section{APÉNDICE 3: Vaciado de datos de la Encuesta sobre Intereses}

\section{Profesionales}

\begin{tabular}{|c|c|c|c|}
\cline { 2 - 4 } \multicolumn{1}{c|}{} & \multicolumn{3}{c|}{ ORIENTACIÓN VOCACIONAL } \\
\cline { 2 - 4 } \multicolumn{1}{c|}{} & $\begin{array}{c}\text { ALUMNAS DECIDIDAS } \\
\text { (1 OPCION) }\end{array}$ & $\begin{array}{c}\text { ALUMNAS CON MAS } \\
\text { DE } 1 \text { OPCION }\end{array}$ & $\begin{array}{c}\text { ALUMNAS NO } \\
\text { DECIDIDAS }\end{array}$ \\
\hline $5 A$ & 7 & 19 & 3 \\
\hline $5 B$ & 11 & 11 & 8 \\
\hline $5 C$ & 16 & 2 & 8 \\
\hline $5 D$ & 18 & 5 & 7 \\
\hline
\end{tabular}

\begin{tabular}{|c|c|c|}
\hline & \multicolumn{2}{|c|}{ CARRERAS } \\
\hline & 1ra OPCIón & $2 \triangleleft a O P C I O ́ N$ \\
\hline Cantidad de Alumnas & 52 & 37 \\
\hline Administración & 10 & 19 \\
\hline Ingenieria & 5 & 10 \\
\hline Psicologia & 6 & 8 \\
\hline Comunicación & 6 & 20 \\
\hline Derecho & 4 & 5 \\
\hline Medicina & 6 & 1 \\
\hline Economía / Finanzas & 3 & 1 \\
\hline Arquitectura & 3 & 6 \\
\hline Publicidad / Marketing & 0 & 8 \\
\hline Educación & 2 & 1 \\
\hline $\begin{array}{c}\text { Negocios } \\
\text { Internacionales }\end{array}$ & 1 & 5 \\
\hline $\begin{array}{c}\text { Artes escénicas / } \\
\text { Teatro }\end{array}$ & 1 & 4 \\
\hline Diseño & 3 & 3 \\
\hline Ciencias Politicas & 1 & 0 \\
\hline Biologia Marina & 1 & 0 \\
\hline Fotografia & 0 & 1 \\
\hline Fisica / Astronomía & 1 & 0 \\
\hline Cinemataografia & 1 & 0 \\
\hline
\end{tabular}

\begin{tabular}{|l|c|}
\hline \multicolumn{2}{|c|}{ UNIVERSIDADES } \\
\hline Universidad & Cantidad de Alumnas \\
\hline Universidad de Lima & 32 \\
\hline UPC & 37 \\
\hline Pacifico & 9 \\
\hline Católica & 5 \\
\hline UCAL & 5 \\
\hline UTEC & 9 \\
\hline Cayetano Heredia & 1 \\
\hline Universidad Agraria & 1 \\
\hline Cientifica del Sur & 3 \\
\hline Universidad de Piura & 4 \\
\hline USIL & 22 \\
\hline San Marcos & 30 \\
\hline $\begin{array}{l}\text { Estudios en el } \\
\text { Extranjero }\end{array}$ & 9 \\
\hline
\end{tabular}




\section{DEPARTAMENTO PSICOPEDAGÓGICO Informe de Orientación Vocacional}

Nombre:

Nro. de Orden:

Sección: 5to

Fecha de informe:

I. Pruebas administradas:

a) Test de preferencias del Temperamento - UPC

b) Test de Talentos - UPC

c) Test de Intereses Profesionales - UPC

d) Test de Aptitudes Mentales PMA

II. Resultados- Interpretación

a) Temperamento:

Brinda información acerca de las características más importantes de la personalidad, a partir de cuatro ejes: Extrovertido-Introvertido, Intuitivo-Sensorial, Racional-Emocional y OrganizadoCasual.

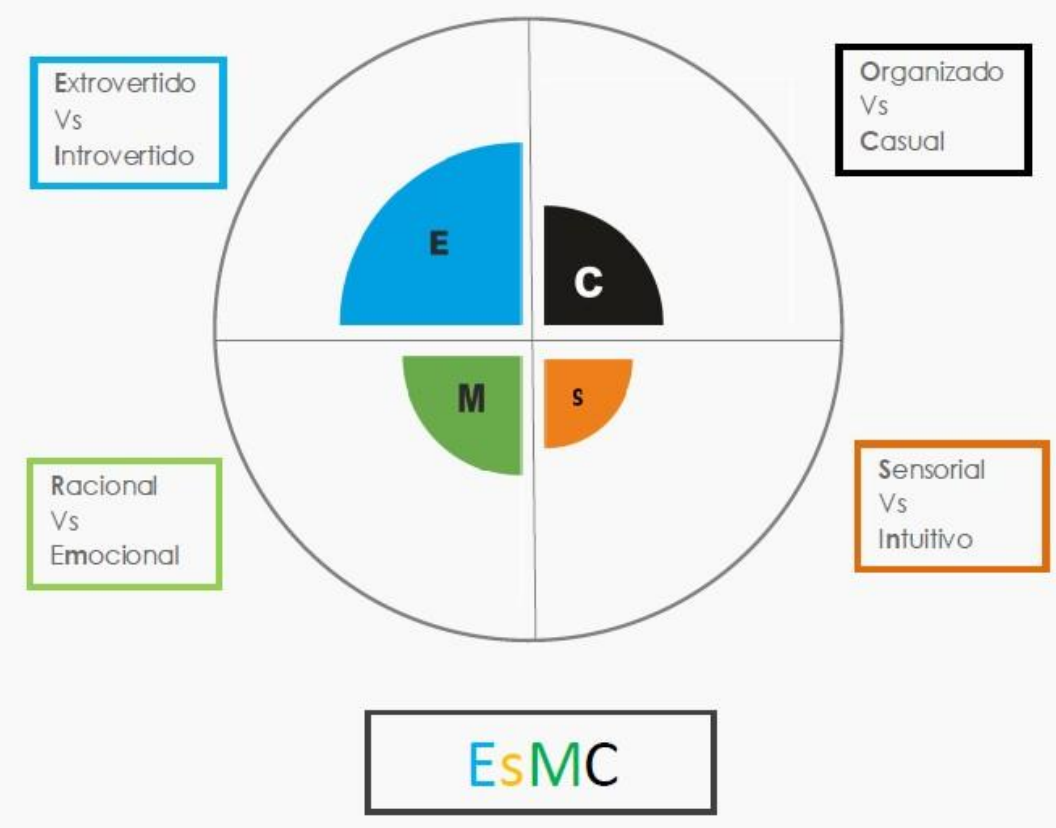

Interpretación:

(Se presentan una descripción general, y luego se especifica lo más resaltante encontrado en cada uno de los ejes, empezando por los que están más desarrollados). 


\section{b) Test de Talentos:}

Herramienta que permite identificar aquellos talentos, es decir, aquello que hace muy bien, en lo que destaca por encima de los demás, y que, además, le genera placer. Algo que, con esfuerzo y perseverancia, pueden llegar a convertirse en nuestras fortalezas.

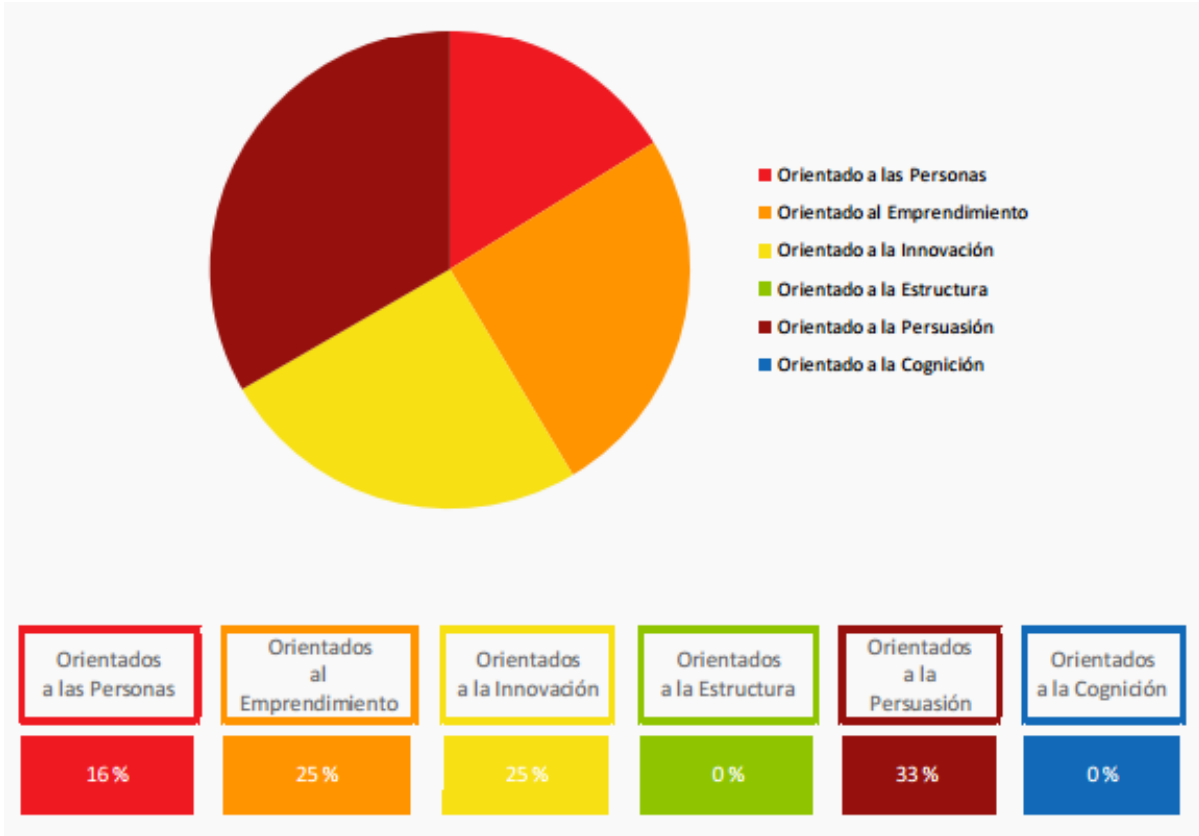

Interpretación:

(Se indican y describen los talentos, empezando por los más destacados y culminando con los menos desarrollados). 


\section{c) Test de Intereses Profesionales}

Se evalúa los intereses profesionales a partir de tres aspectos: el gusto por realizar alguna actividad en su vida profesional, las habilidades que tiene para aprender a realizar esta actividad y la satisfacción que cree que le brindaría esta actividad como parte de tu profesión.

Tabla de resultados:

\begin{tabular}{|c|c|c|c|c|}
\hline \multirow{2}{*}{ ÁREA DE INTERÉs } & \multicolumn{4}{|c|}{ Puntaje } \\
\hline & \begin{tabular}{|l|} 
Valor \\
\end{tabular} & Bajo & Medio & Allio \\
\hline ADMINISTLACION & 98 & & & \\
\hline AGRARIA & 22 & & & \\
\hline AvTiSTICA & 98 & & & \\
\hline COMUNICACIÓN & 39 & & & \\
\hline CONSTRUCCIÓN & 16 & & & \\
\hline CUUMAMA & 98 & & & \\
\hline DEPORTIA & 5 & & & \\
\hline DISENOO & 31 & & & \\
\hline FIMANCIERA & 79 & & & \\
\hline INFORMÁnCA & 3 & & & \\
\hline sunioico & 96 & & & \\
\hline MARKEIINO & 98 & & & \\
\hline MECÁNICO/ELECTRÓNICA & 8 & & & \\
\hline Manera & 28 & & & \\
\hline PEDACOCIA & 26 & & & \\
\hline SALUD & 94 & & & \\
\hline SOCIAL & 97 & & & \\
\hline TEADUCCIÓN & 73 & & & \\
\hline Tunsmo & 98 & & & \\
\hline
\end{tabular}

$\underline{\text { Interpretación }}$

(Indicar áreas de mayor interés). 
Cuadro de carreras:

\begin{tabular}{|c|c|c|}
\hline \multicolumn{2}{|r|}{ Área } & Carreras Asociadas \\
\hline Antisnca & $\begin{array}{l}\text { interds por la producción o } \\
\text { participoción en presentaciones } \\
\text { ortísticos de teotio, dorna o } \\
\text { música. }\end{array}$ & $\begin{array}{l}\text { Antes Escénicos } \\
\text { Antista Proflesiond } \\
\text { Donza } \\
\text { Mistico }\end{array}$ \\
\hline MARKEIINO & $\begin{array}{l}\text { Inteds en la identificación de los } \\
\text { necesidades de los conbumidores } \\
\text { para ofteceries productos y } \\
\text { iervicios competithas en el } \\
\text { mercodo. }\end{array}$ & $\begin{array}{l}\text { Administración y markating } \\
\text { Comunicación e imagen } \\
\text { Empresarid } \\
\text { Comunicacion y Markering } \\
\text { Publicidad } \\
\text { Comunicación y Publicidad }\end{array}$ \\
\hline CUUNARIA & 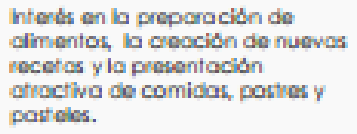 & $\begin{array}{l}\text { Arte culinario } \\
\text { Castionomia } \\
\text { Cationomia y Gestisn de } \\
\text { Restarantes }\end{array}$ \\
\hline ADMINISTEACION & 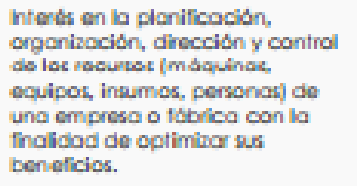 & 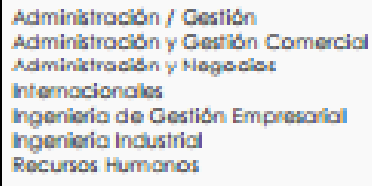 \\
\hline Tunsmo & 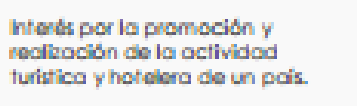 & $\begin{array}{l}\text { Ecoluriamo } \\
\text { Turismo } \\
\text { Hotelefía } \\
\text { Hotelefí y Administuocion }\end{array}$ \\
\hline
\end{tabular}

d) Aptitudes Mentales- PMA: Se evalúan 5 áreas/Factores

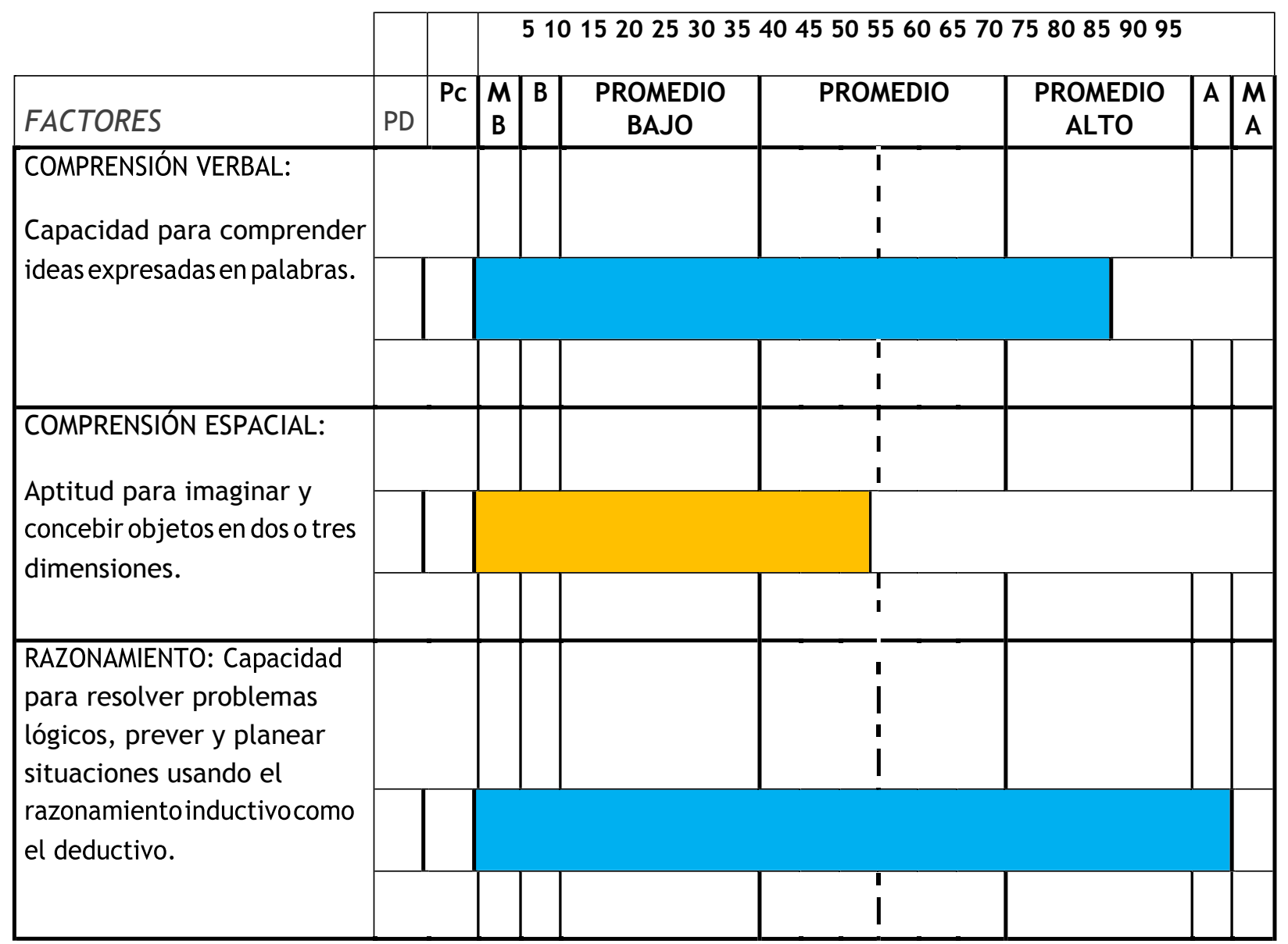




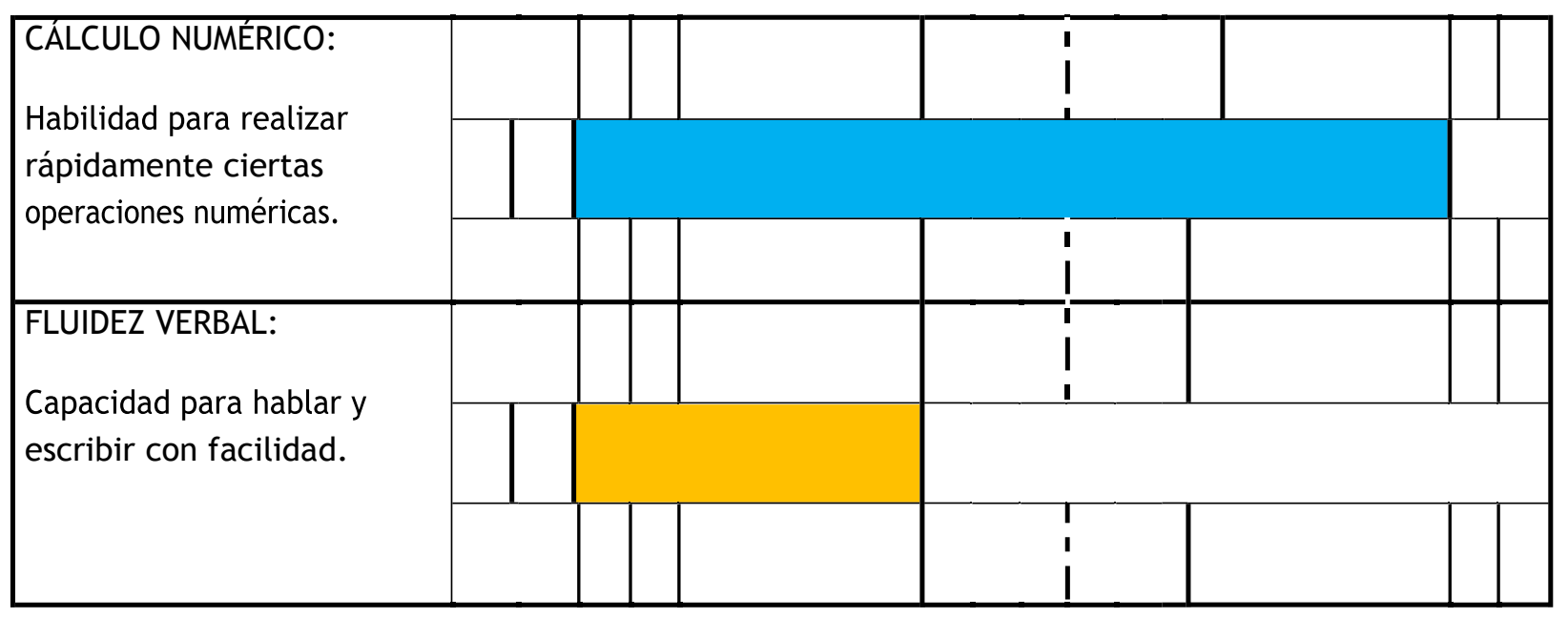

\section{Comentario:}

